\title{
Complex Spacetime Frame: Four-Vector Identities and Tensors
}

\author{
Joseph Akeyo Omolo \\ Department of Physics and Materials Science, Maseno University, Maseno, Kenya \\ Email: ojakeyo04@yahoo.co.uk
}

Received 29 July 2014; revised 29 August 2014; accepted 15 September 2014

Copyright (C) 2014 by author and Scientific Research Publishing Inc.

This work is licensed under the Creative Commons Attribution International License (CC BY). http://creativecommons.org/licenses/by/4.0/

(c) (i) Open Access

\section{Abstract}

This paper provides derivation of some basic identities for complex four-component vectors defined in a complex four-dimensional spacetime frame specified by an imaginary temporal axis. The resulting four-vector identities take exactly the same forms of the standard vector identities established in the familiar three-dimensional space, thereby confirming the consistency of the definition of the complex four-vectors and their mathematical operations in the general complex spacetime frame. Contravariant and covariant forms have been defined, providing appropriate definitions of complex tensors, which point to the possibility of reformulating differential geometry within a spacetime frame.

\section{Keywords}

Complex Spacetime Frame, Four-Vector Identities, Contravariant and Covariant Forms, Complex Tensors

\section{Introduction}

In a recent derivation [1], the present author identified the unit wave vector $\hat{k}$ to be the temporal unit vector within four-dimensional spacetime frame. The temporal direction is specified as an imaginary axis with unit vector $i \hat{k}$, where $i=\sqrt{-1}$ is the imaginary number. General spacetime frame is then defined as a complex four-dimensional coordinate system spanned by the temporal unit vector $i \hat{k}$ and the three mutually perpendicular spatial unit vectors $\hat{x}, \hat{y}, \hat{z}$ specifying the $x, y, z$ axes, respectively. We take the temporal unit vector $\hat{k}$ to have general orientation relative to the spatial unit vectors according to

$$
\hat{k} \cdot \hat{x} \neq 0, \quad \hat{k} \cdot \hat{y} \neq 0, \quad \hat{k} \cdot \hat{z} \neq 0
$$

The usual assumption, implicit in conventional four-vector mathematics that the temporal axis is perpendicu- 
lar to all the three mutually perpendicular spatial axes, with $\hat{k} \cdot \hat{x}=0, \hat{k} \cdot \hat{y}=0, \hat{k} \cdot \hat{z}=0$, may occur only as a special case to be specified. The basic elements of the complex spacetime frame are complex four-component vectors, which we generally call four-vectors. We define a general four-vector $V$ in the form

$$
V=V_{k} \hat{k}+\boldsymbol{V}=V_{k} \hat{k}+V_{x} \hat{x}+V_{y} \hat{y}+V_{z} \hat{z}
$$

with the imaginary temporal component $V_{k}$ defined by

$$
V_{k}=-i c \phi, \quad \phi=\phi(\boldsymbol{r}, t)
$$

where $\phi$ is a scalar quantity specifying the nature of the temporal component of the four-vector. As usual, $t$ denotes time. In general, the temporal component of each four-vector occurs along the imaginary temporal axis, $i \hat{k}$, multiplied by a factor $-c$, where $c$ is the speed of light. We follow standard convention denoting fourvectors by uppercase letters, e.g., $V$, while the usual three-component spatial vectors are denoted with arrows over letter symbols, e.g., $\boldsymbol{r}$.

According to the general definition in Equations (2a)-(2b), the spacetime displacement four-vector $X$ takes the form

$$
X=x_{k} \hat{k}+\boldsymbol{r}=x_{k} \hat{k}+x \hat{x}+y \hat{y}+z \hat{z}, \quad x_{k}=-i c t
$$

while the corresponding event interval $\mathrm{d} X$ between two neighboring spacetime points, takes the form

$$
\mathrm{d} X=\mathrm{d} x_{k} \hat{k}+\mathrm{d} \boldsymbol{r}=\mathrm{d} x_{k} \hat{k}+\mathrm{d} x \hat{x}+\mathrm{d} y \hat{y}+\mathrm{d} z \hat{z}, \quad \mathrm{~d} x_{k}=-i c \mathrm{~d} t
$$

The spacetime derivative four-vector, $\nabla$ is defined as

$$
\nabla=\frac{\partial}{\partial x_{k}} \hat{k}+\nabla=\frac{i}{c} \frac{\partial}{\partial t} \hat{k}+\nabla
$$

where $x_{k}=-i c t$ and $\nabla$ is the usual three-component spatial gradient vector defined by

$$
\nabla=\frac{\partial}{\partial x} \hat{x}+\frac{\partial}{\partial y} \hat{y}+\frac{\partial}{\partial z} \hat{z}
$$

Interpreting $\nabla$ in Equation (4a) in terms of the general four-vector $V$ in Equations (2a)-(2b) gives

$$
V=\nabla \Rightarrow V_{k}=\frac{i}{c} \frac{\partial}{\partial t}, \quad \phi=-\frac{1}{c^{2}} \frac{\partial}{\partial t}, \quad V=\nabla
$$

We observe that the concept of imaginary temporal axis developed here, represents a rediscovery of the idea of imaginary time first introduced independently by Poincare [2], Lorentz [3] and Einstein [4] in their original theories of electrodynamics or special relativity in a four-dimensional spacetime frame. These authors did not identify the temporal unit vector and therefore could not completely specify the complex spacetime frame and develop the full mathematical operations using complex four-vectors in the manner presented in this paper.

\section{Mathematical Operations with Four-Vectors}

The general four-component vector form in Equation (2a) with all unit vectors specified allows us to carry out four-vector mathematical operations in the complex spacetime frame in exactly the same manner as the standard mathematical operations with the familiar three-component vectors in three-dimensional space.

In developing the mathematical operations in general form, we shall take the temporal unit vector $\hat{k}$ to be of general orientation relative to the spatial unit vectors $\hat{x}, \hat{y}, \hat{z}$, satisfying the conditions in Equation (1). We denote four-vectors with arrows, while the three-component spatial vectors are written in boldface as stated above. We use two general four-vectors $V$ and $U$ defined by

$$
V=V_{k} \hat{k}+\boldsymbol{V}, \quad V_{k}=-i c \phi, \quad U=U_{k} \hat{k}+\boldsymbol{U}, \quad U_{k}=-i c \varphi
$$

to develop the mathematical operations with four-vectors. The basic mathematical operations are essentially addition, subtraction, dot product, cross product, divergence and curl.

\subsection{Addition and Subtraction}

Four-vector addition and subtraction is straightforward, taking the form 


$$
W=U \pm V=\left(U_{k} \pm V_{k}\right) \hat{k}+(\boldsymbol{U} \pm \boldsymbol{V})
$$

\subsection{The Dot Product}

The dot product of the four-vectors $U$ and $V$ is obtained as

$$
U \cdot V=\left(U_{k} \hat{k}+\boldsymbol{U}\right) \cdot\left(V_{k} \hat{k}+\boldsymbol{V}\right)
$$

which we expand term by term, maintaining the order of components in the products and then substitute $U_{k}=-i c \varphi, V_{k}=-i c \phi$ from Equation (5), to obtain the dot product in the final form

$$
U \cdot V=\boldsymbol{U} \cdot \boldsymbol{V}-c^{2} \varphi \phi-i c \hat{k} \cdot(\varphi \boldsymbol{V}+\boldsymbol{U} \phi)
$$

\subsection{The Cross Product}

The cross product of the four-vectors $U$ and $V$ is obtained as

$$
U \times V=\left(U_{k} \hat{k}+U\right) \times\left(V_{k} \hat{k}+V\right)
$$

which we expand term by term, using $\hat{k} \times \hat{k}=0, \boldsymbol{U} \times \hat{k}=-\hat{k} \times \boldsymbol{U}$ and then substitute $U_{k}=-i c \varphi, V_{k}=-i c \phi$ from Equation (5) to obtain the cross product in the final form

$$
U \times V=\boldsymbol{U} \times \boldsymbol{V}-i c \hat{k} \times(\varphi \boldsymbol{V}-\boldsymbol{U} \phi)
$$

\subsection{Divergence of a Four-Vector}

Setting $U$ equal to the spacetime derivative four-vector $\nabla$ according to

$$
U=\nabla=\frac{i}{c} \frac{\partial}{\partial t} \hat{k}+\nabla=-i c\left(-\frac{1}{c^{2}} \frac{\partial}{\partial t}\right) \hat{k}+\nabla=-i c \varphi \hat{k}+\boldsymbol{U}
$$

with

$$
\varphi=-\frac{1}{c^{2}} \frac{\partial}{\partial t}, \quad U=\nabla
$$

in the general four-vector dot product obtained in Equation (7b), we obtain the divergence of a general fourvector $V$ in the final form

$$
\nabla \cdot V=\frac{\partial \phi}{\partial t}+\nabla \cdot V+i \hat{k} \cdot\left(\frac{1}{c} \frac{\partial \boldsymbol{V}}{\partial t}-\nabla(c \phi)\right)
$$

\subsection{Curl of a Four-Vector}

Setting $U$ equal to the spacetime derivative four-vector $\nabla$ according to Equations (9a), (9b) in the general four-vector cross product obtained in Equation (8b), we obtain the curl of a general four-vector $V$ in the final form

$$
\nabla \times V=\nabla \cdot \boldsymbol{V}-i\left(\frac{1}{c} \frac{\partial \boldsymbol{V}}{\partial t}+\nabla(c \phi)\right) \times \hat{k}
$$

\section{Four-Vector Identities}

We now derive some basic four-vector identities in complex four-dimensional spacetime frame, which generalize standard vector identities in three-dimensional Euclidean space [5] [6].

\subsection{Curl of Gradient Four-Vector}

A gradient four-vector $\nabla \Phi$ generated through application of the spacetime derivative four-vector $\nabla$ on a scalar function $\Phi$ is obtained as 


$$
\nabla \Phi=\left(\frac{i}{c} \frac{\partial}{\partial t} \hat{k}+\nabla\right) \Phi=-i c\left(-\frac{1}{c^{2}} \frac{\partial \Phi}{\partial t}\right) \hat{k}+\nabla \Phi
$$

Setting the general four-vector $V$ equal to the gradient four-vector according to

$$
V=\nabla \Phi=-i c\left(-\frac{1}{c^{2}} \frac{\partial \Phi}{\partial t}\right) \hat{k}+\nabla \Phi=-i c \phi \hat{k}+\boldsymbol{V}
$$

with

$$
\phi=-\frac{1}{c^{2}} \frac{\partial \Phi}{\partial t}, \quad \boldsymbol{V}=\nabla \Phi
$$

in the general curl of a four-vector obtained in Equation (10), we obtain the curl of a gradient four-vector $\nabla \Phi$ in the form

$$
\nabla \times \nabla \Phi=\nabla \times \nabla \Phi-i\left(\frac{1}{c} \frac{\partial \nabla \Phi}{\partial t}-\nabla\left(\frac{1}{c} \frac{\partial \Phi}{\partial t}\right)\right) \times \hat{k}
$$

which on using the standard three-dimensional space vector analysis results

$$
\nabla \times \nabla \Phi=0, \quad \frac{1}{c} \frac{\partial \nabla \Phi}{\partial t}=\nabla\left(\frac{1}{c} \frac{\partial \Phi}{\partial t}\right)
$$

gives the final result

$$
\nabla \times \nabla \Phi=0
$$

This shows that the curl of a gradient four-vector vanishes. This four-vector identity generalizes the corresponding vector identity in standard three-dimensional Euclidean space [5] [6] given in the first part of Equation (11e).

\subsection{Divergence of Curl of a Four-Vector}

Taking the divergence of the curl of the general four-vector $V$ in Equation (10), we obtain

$$
\nabla \cdot \nabla \times V=\left(\frac{i}{c} \frac{\partial}{\partial t} \hat{k}+\nabla\right) \cdot\left(\nabla \times \boldsymbol{V}-i\left(\frac{1}{c} \frac{\partial \boldsymbol{V}}{\partial t}+\nabla(c \phi)\right) \times \hat{k}\right)
$$

which on expanding term by term becomes

$$
\nabla \cdot \nabla \times V=\frac{i}{c} \frac{\partial}{\partial t} \hat{k} \cdot \nabla \times \boldsymbol{V}+\frac{1}{c} \frac{\partial}{\partial t} \hat{k} \cdot\left(\frac{1}{c} \frac{\partial \boldsymbol{V}}{\partial t}+\nabla(c \phi)\right) \times \hat{k}+\nabla \cdot \nabla \times \boldsymbol{V}-i \nabla \cdot\left(\frac{1}{c} \frac{\partial \boldsymbol{V}}{\partial t}+\nabla(c \phi)\right) \times \hat{k}
$$

Applying standard three-dimensional space vector analysis results

$$
\nabla \cdot \nabla \times \boldsymbol{V}=0, \quad \hat{k} \cdot\left(\frac{1}{c} \frac{\partial \boldsymbol{V}}{\partial t}+\nabla(c \phi)\right) \times \hat{k}=0
$$

we express Equation (13b) in the form

$$
\nabla \cdot \nabla \times V=i\left\{\hat{k} \cdot\left(\nabla \times \frac{1}{c} \frac{\partial \boldsymbol{V}}{\partial t}\right)-\nabla \cdot\left(\frac{1}{c} \frac{\partial \boldsymbol{V}}{\partial t}+\nabla(c \phi)\right) \times \hat{k}\right\}
$$

Application of standard three-dimensional space vector identity

$$
\nabla \cdot(\boldsymbol{Q} \times \boldsymbol{R})=\boldsymbol{R} \cdot(\boldsymbol{\nabla} \times \boldsymbol{Q})-\boldsymbol{Q} \cdot(\boldsymbol{\nabla} \times \boldsymbol{R})
$$

gives

$$
\nabla \cdot\left(\frac{1}{c} \frac{\partial \boldsymbol{V}}{\partial t}+\nabla(c \phi)\right) \times \hat{k}=\hat{k} \cdot\left(\nabla \times\left(\frac{1}{c} \frac{\partial \boldsymbol{V}}{\partial t}+\nabla(c \phi)\right)\right)-\left(\frac{1}{c} \frac{\partial \boldsymbol{V}}{\partial t}+\nabla(c \phi)\right) \cdot(\nabla \times \hat{k})
$$

which on using 


$$
\nabla \times \nabla(c \phi)=0, \quad \nabla \times \hat{k}=0
$$

takes the final form

$$
\nabla \cdot\left(\frac{1}{c} \frac{\partial \boldsymbol{V}}{\partial t}+\nabla(c \phi)\right) \times \hat{k}=\hat{k} \cdot\left(\nabla \times \frac{1}{c} \frac{\partial \boldsymbol{V}}{\partial t}\right)
$$

Substituting Equation (13h) into Equation (13d) gives the final result

$$
\nabla \cdot \nabla \times V=0
$$

This shows that the divergence of curl of a four-vector vanishes. This four-vector identity generalizes the corresponding vector identity in standard three-dimensional Euclidean space [5] [6] given in the first part of Equation (13c).

\subsection{General Vanishing Four-Vector Dot Product: $U \cdot(U \times V)$}

The important identity on the vanishing of the divergence of curl of a four-vector in Equation (14) can be generalized by taking the dot product of the four-vector $U$ and the cross product of the four-vectors $U$ and $V$ which on using the general result in Equation (8b) is obtained as

$$
U \cdot(U \times V)=\left(U_{k} \hat{k}+\boldsymbol{U}\right) \cdot\left\{\boldsymbol{U} \times \boldsymbol{V}+\hat{k} \times\left(U_{k} \boldsymbol{V}-\boldsymbol{U} V_{k}\right)\right\}
$$

which we expand term by term and use standard three-dimensional space vector identities

$$
\boldsymbol{U} \cdot(\boldsymbol{U} \times \boldsymbol{V})=0, \quad \hat{k} \cdot\left\{\hat{k} \times\left(U_{k} \boldsymbol{V}-\boldsymbol{U} V_{k}\right)\right\}=0
$$

to obtain

$$
U \cdot(U \times V)=\hat{k} \cdot\left(U_{k} \boldsymbol{U} \times \boldsymbol{V}\right)+\boldsymbol{U} \cdot\left\{\hat{k} \times\left(U_{k} \boldsymbol{V}-\boldsymbol{U} V_{k}\right)\right\}
$$

Applying a three-dimensional space vector identity

$$
\boldsymbol{U} \cdot\left\{\hat{k} \times\left(U_{k} \boldsymbol{V}-\boldsymbol{U} V_{k}\right)\right\}=\hat{k} \cdot\left\{\left(U_{k} \boldsymbol{V}-\boldsymbol{U} V_{k}\right) \times \boldsymbol{U}\right\}
$$

and using

$$
\boldsymbol{U} V_{k} \times \boldsymbol{U}=V_{k} \boldsymbol{U} \times \boldsymbol{U}=0
$$

gives

$$
\boldsymbol{U} \cdot\left\{\hat{k} \times\left(U_{k} \boldsymbol{V}-\boldsymbol{U} V_{k}\right)\right\}=-\hat{k} \cdot\left(U_{k} \boldsymbol{U} \times \boldsymbol{V}\right)
$$

which we substitute into Equation (15c) to obtain the final result

$$
U \cdot(U \times V)=0
$$

This result generalizes the divergence of curl of a four-vector obtained in Equation (14). It is a generalization of the corresponding vector identity in standard three-dimensional Euclidean space [5] [6] given in the first part of Equation (15b).

\subsection{Divergence and Curl of $f V$}

For a scalar function $f(r, t)$, we use the definitions of $V$ and $\nabla$ from Equations (2a) and (4a) to obtain

$$
\nabla \cdot(f V)=\left(\frac{i}{c} \frac{\partial}{\partial t} \hat{k}+\nabla\right) \cdot\left\{f\left(V_{k} \hat{k}+V\right)\right\}, \quad \nabla \times(f V)=\left(\frac{i}{c} \frac{\partial}{\partial t} \hat{k}+\nabla\right) \times\left\{f\left(V_{k} \hat{k}+V\right)\right\}
$$

Expanding these term by term gives

$$
\nabla \cdot(f V)=\left(\frac{i}{c} \frac{\partial f}{\partial t} \hat{k}+\nabla f\right) \cdot V_{k} \hat{k}+\left(\frac{i}{c} \frac{\partial f}{\partial t} \hat{k}+\nabla f\right) \cdot V+f\left(\frac{i}{c} \frac{\partial}{\partial t} \hat{k}+\nabla\right) \cdot V_{k} \hat{k}+f\left(\frac{i}{c} \frac{\partial}{\partial t} \hat{k}+\nabla\right) \cdot V
$$




$$
\nabla \times(f V)=\left(\frac{i}{c} \frac{\partial f}{\partial t} \hat{k}+\nabla f\right) \times V_{k} \hat{k}+\left(\frac{i}{c} \frac{\partial f}{\partial t} \hat{k}+\nabla f\right) \times \boldsymbol{V}+f\left(\frac{i}{c} \frac{\partial f}{\partial t} \hat{k}+\nabla\right) \times V_{k} \hat{k}+f\left(\frac{i}{c} \frac{\partial f}{\partial t} \hat{k}+\nabla\right) \times \boldsymbol{V}
$$

which we reorganize to obtain the four-vector identities

$$
\nabla \cdot(f V)=\nabla f \cdot V+f \nabla \cdot V, \quad \nabla \times(f V)=\nabla f \times V+f \nabla \times V
$$

These four-vector identities generalize the corresponding vector identities in standard three-dimensional Euclidean space [5] [6].

\subsection{Divergence of Four-Vector Cross Product: $\nabla \cdot(U \times V)$}

We use the general form of the curl of a four-vector from equation (10) to obtain

$$
\begin{aligned}
& U \cdot(\nabla \times V)=\boldsymbol{U} \cdot(\boldsymbol{\nabla} \times \boldsymbol{V})-i \hat{k} \cdot\left\{c \varphi \boldsymbol{\nabla} \times \boldsymbol{V}+\left(\frac{1}{c} \frac{\partial \boldsymbol{V}}{\partial t}+\nabla(c \phi)\right) \times \boldsymbol{U}\right\} \\
& V \cdot(\nabla \times U)=\boldsymbol{V} \cdot(\boldsymbol{\nabla} \times \boldsymbol{U})-i \hat{k} \cdot\left\{c \phi \boldsymbol{\nabla} \times \boldsymbol{U}+\left(\frac{1}{c} \frac{\partial \boldsymbol{U}}{\partial t}+\nabla(c \varphi)\right) \times \boldsymbol{V}\right\}
\end{aligned}
$$

after applying standard three-dimensional space vector identities

$$
\begin{gathered}
\hat{k} \cdot\left(\frac{1}{c} \frac{\partial \boldsymbol{V}}{\partial t}+\nabla(c \phi)\right) \times \hat{k}=0, \quad \hat{k} \cdot\left(\frac{1}{c} \frac{\partial \boldsymbol{U}}{\partial t}+\nabla(c \varphi)\right) \times \hat{k}=0 \\
\boldsymbol{U} \cdot\left(\frac{1}{c} \frac{\partial \boldsymbol{V}}{\partial t}+\nabla(c \phi)\right) \times \hat{k}=\hat{k} \cdot\left(\frac{1}{c} \frac{\partial \boldsymbol{V}}{\partial t}+\nabla(c \phi)\right) \times \boldsymbol{U} \\
\boldsymbol{V} \cdot\left(\frac{1}{c} \frac{\partial \boldsymbol{U}}{\partial t}+\nabla(c \varphi)\right) \times \hat{k}=\hat{k} \cdot\left(\frac{1}{c} \frac{\partial \boldsymbol{U}}{\partial t}+\nabla(c \varphi)\right) \times \boldsymbol{V}
\end{gathered}
$$

Subtracting Equation (17b) from Equation (17a) and applying standard three-dimensional vector identities, together with appropriate rules of differentiation of vector products, we obtain the final result

$$
V \cdot(\nabla \times U)-U \cdot(\nabla \times V)=\nabla \cdot(\boldsymbol{U} \times \boldsymbol{V})+i \hat{k} \cdot\left\{\frac{1}{c} \frac{\partial}{\partial t}(\boldsymbol{U} \times \boldsymbol{V})+\nabla \times(c \varphi \boldsymbol{V})-\nabla \times(c \phi \boldsymbol{U})\right\}
$$

We now use the four-vector cross product from Equation (8b) to obtain

$$
\nabla \cdot(U \times V)=\left(\frac{i}{c} \frac{\partial}{\partial t} \hat{k}+\nabla\right) \cdot(\boldsymbol{U} \times \boldsymbol{V}-i c \hat{k} \times(\varphi \boldsymbol{V}-\boldsymbol{U} \phi))
$$

which we expand as appropriate and apply standard three-dimensional space vector identities

$$
\hat{k} \cdot \hat{k} \times(\varphi \boldsymbol{V}-\boldsymbol{U} \phi)=0, \quad \nabla \cdot \hat{k} \times(\varphi \boldsymbol{V}-\boldsymbol{U} \phi)=-\hat{k} \cdot \nabla \times(\varphi \boldsymbol{V}-\boldsymbol{U} \phi)
$$

to obtain the final result

$$
\nabla \cdot(U \times V)=\nabla \cdot(\boldsymbol{U} \times \boldsymbol{V})+i \hat{k} \cdot\left\{\frac{1}{c} \frac{\partial}{\partial t}(\boldsymbol{U} \times \boldsymbol{V})+\nabla \times(c \varphi \boldsymbol{V})-\nabla \times(c \phi \boldsymbol{U})\right\}
$$

Substituting Equation (17f) into Equation (18c) gives the four-vector identity

$$
\nabla \cdot(U \times V)=V \cdot(\nabla \times U)-U \cdot(\nabla \times V)
$$

This four-vector identity generalizes the corresponding vector identity in standard three-dimensional Euclidean space [5] [6] given earlier in Equation (13e).

\subsection{The Curl of a Four-Vector: $\nabla \times(\nabla \times V)$}

Let us start by taking the four-vector curl of the curl of the general complex four-vector in Equation (10) to obtain 


$$
\nabla \times(\nabla \times V)=\left(\frac{i}{c} \frac{\partial}{\partial t} \hat{k}+\nabla\right) \times\left(\nabla \times V+i \hat{k} \times\left(\frac{1}{c} \frac{\partial \boldsymbol{V}}{\partial t}+\nabla(c \Lambda)\right)\right)
$$

which we on expansion takes the form

$$
\nabla \times(\nabla \times V)=i \hat{k} \times\left(\nabla \times \frac{1}{c} \frac{\partial \boldsymbol{V}}{\partial t}\right)-\frac{1}{c} \frac{\partial}{\partial t} \hat{k} \times\left\{\hat{k} \times\left(\frac{1}{c} \frac{\partial \boldsymbol{V}}{\partial t}+\nabla(c \Lambda)\right)\right\}+\nabla \times(\nabla \times \boldsymbol{V})+i \nabla \times\left\{\hat{k} \times\left(\frac{1}{c} \frac{\partial \boldsymbol{V}}{\partial t}+\nabla(c \Lambda)\right)\right\}
$$

Next, we take the four-vector gradient of the divergence of the general complex four-vector in Equation (9c) to obtain

$$
\nabla(\nabla \times V)=\left(\frac{i}{c} \frac{\partial}{\partial t} \hat{k}+\nabla\right)\left(\frac{\partial \Lambda}{\partial t}+\nabla \cdot V+i \hat{k} \cdot\left(\frac{1}{c} \frac{\partial \boldsymbol{V}}{\partial t}-\nabla(c \Lambda)\right)\right)
$$

which we expand in the form

$$
\nabla(\nabla \cdot V)=\frac{i}{c} \frac{\partial}{\partial t} \hat{k}\left(\frac{\partial \Lambda}{\partial t}+\nabla \cdot \boldsymbol{V}\right)-\frac{1}{c} \frac{\partial}{\partial t} \hat{k}\left\{\hat{k} \cdot\left(\frac{1}{c} \frac{\partial \boldsymbol{V}}{\partial t}-\nabla(c \Lambda)\right)\right\}+\nabla\left(\frac{\partial \Lambda}{\partial t}+\nabla \cdot \boldsymbol{V}\right)+i \nabla\left\{\hat{k} \cdot\left(\frac{1}{c} \frac{\partial \boldsymbol{V}}{\partial t}-\nabla(c \Lambda)\right)\right\}(1
$$

We apply standard three-dimensional Euclidean space vector identities giving

$$
\begin{gathered}
\hat{k} \times\left\{\hat{k} \cdot\left(\frac{1}{c} \frac{\partial \boldsymbol{V}}{\partial t}-\nabla(c \Lambda)\right)\right\}=\hat{k}\left\{\hat{k} \cdot\left(\frac{1}{c} \frac{\partial \boldsymbol{V}}{\partial t}-\nabla(c \Lambda)\right)\right\}-\left(\frac{1}{c} \frac{\partial \boldsymbol{V}}{\partial t}-\nabla(c \Lambda)\right) \frac{1}{c} \frac{\partial}{\partial t} \hat{k}\left\{\hat{k} \cdot\left(\frac{1}{c} \frac{\partial \boldsymbol{V}}{\partial t}-\nabla(c \Lambda)\right)\right\} \\
=\frac{1}{c} \frac{\partial}{\partial t} \hat{k} \times\left\{\hat{k} \cdot\left(\frac{1}{c} \frac{\partial \boldsymbol{V}}{\partial t}-\nabla(c \Lambda)\right)\right\}+\frac{1}{c} \frac{\partial}{\partial t}\left(\frac{1}{c} \frac{\partial \boldsymbol{V}}{\partial t}-\nabla(c \Lambda)\right) . \\
\vec{\nabla}\left\{\hat{k} \cdot\left(\frac{1}{c} \frac{\partial \boldsymbol{V}}{\partial t}-\nabla(c \Lambda)\right)\right\}=\hat{k} \times\left(\nabla \times \frac{1}{c} \frac{\partial \boldsymbol{V}}{\partial t}\right)+(\hat{k} \cdot \nabla)\left(\frac{1}{c} \frac{\partial \boldsymbol{V}}{\partial t}-\nabla(c \Lambda)\right) \\
\nabla(\nabla \cdot \boldsymbol{V})=\nabla \times(\nabla \cdot \boldsymbol{V})+\nabla^{2} \boldsymbol{V}
\end{gathered}
$$

which we substitute into Equation (19d) as appropriate to obtain the final form

$$
\begin{aligned}
\nabla(\nabla \cdot V)= & \frac{i}{c} \frac{\partial}{\partial t} \hat{k}\left(\frac{\partial \Lambda}{\partial t}+\nabla \cdot \boldsymbol{V}\right)+\nabla \frac{\partial \Lambda}{\partial t}+\nabla \times(\nabla \cdot \boldsymbol{V})+\nabla^{2} \boldsymbol{V}-\frac{1}{c} \frac{\partial}{\partial t} \hat{k} \times\left\{\hat{k} \cdot\left(\frac{1}{c} \frac{\partial \boldsymbol{V}}{\partial t}-\nabla(c \Lambda)\right)\right\} \\
& -\frac{1}{c} \frac{\partial}{\partial t}\left(\frac{1}{c} \frac{\partial \boldsymbol{V}}{\partial t}-\nabla(c \Lambda)\right)+i \hat{k} \times\left(\nabla \times \frac{1}{c} \frac{\partial \boldsymbol{V}}{\partial t}\right)+i(\hat{k} \cdot \nabla)\left(\frac{1}{c} \frac{\partial \boldsymbol{V}}{\partial t}-\nabla(c \Lambda)\right) .
\end{aligned}
$$

Subtracting Equation (19b) from Equation (19h) and using standard three-dimensional Euclidean space vector identities giving

$$
\begin{gathered}
\nabla \times\left\{\hat{k} \cdot\left(\frac{1}{c} \frac{\partial \boldsymbol{V}}{\partial t}+\nabla(c \Lambda)\right)\right\}=\left\{\nabla \cdot\left(\frac{1}{c} \frac{\partial \boldsymbol{V}}{\partial t}+\nabla(c \Lambda)\right)\right\} \hat{k}-(\hat{k} \cdot \nabla)\left(\frac{1}{c} \frac{\partial \boldsymbol{V}}{\partial t}+\nabla(c \Lambda)\right) \\
\hat{k} \times(\hat{k} \times \nabla(c \Lambda))=(\hat{k} \cdot \nabla(c \Lambda)) \hat{k}-\nabla(c \Lambda)
\end{gathered}
$$

we obtain

$$
\begin{aligned}
& \nabla(\nabla \cdot V)-\nabla \times(\nabla \times V) \\
& \quad=\frac{i}{c} \frac{\partial}{\partial t} \hat{k}\left(\frac{\partial \Lambda}{\partial t}+\nabla \cdot \boldsymbol{V}\right)+\nabla^{2} \boldsymbol{V}-\frac{1}{c^{2}} \frac{\partial^{2} \boldsymbol{V}}{\partial t^{2}}+\frac{2}{c} \frac{\partial}{\partial t}(\hat{k} \cdot \nabla)(c \Lambda \hat{k}+i \boldsymbol{V})-i\left\{\nabla \cdot\left(\frac{1}{c} \frac{\partial \boldsymbol{V}}{\partial t}+\nabla(c \Lambda)\right)\right\} \hat{k} .
\end{aligned}
$$

which on reorganizing

$$
\left\{\nabla \cdot\left(\frac{1}{c} \frac{\partial \boldsymbol{V}}{\partial t}+\nabla(c \Lambda)\right)\right\} \hat{k}=\left\{\frac{1}{c} \frac{\partial(\boldsymbol{\nabla} \cdot \boldsymbol{V})}{\partial t}+\nabla^{2}(c \Lambda)\right\} \hat{k}, \quad c \Lambda \hat{k}+i \boldsymbol{V}=i(-i c \Lambda \hat{k}+\boldsymbol{V})=i V
$$

takes the form 


$$
\nabla(\nabla \cdot V)-\nabla \times(\nabla \times V)=i\left(\frac{1}{c^{2}} \frac{\partial^{2}(c \Lambda)}{\partial t^{2}}-\nabla^{2}(c \Lambda)\right) \hat{k}+\left(\nabla^{2} \boldsymbol{V}-\frac{1}{c^{2}} \frac{\partial^{2} \boldsymbol{V}}{\partial t^{2}}\right)+2 i(\hat{k} \cdot \nabla) \frac{1}{c} \frac{\partial V}{\partial t}
$$

We easily obtain

$$
i\left(\frac{1}{c^{2}} \frac{\partial^{2}(c \Lambda)}{\partial t^{2}}-\nabla^{2}(c \Lambda)\right) \hat{k}+\left(\nabla^{2} \boldsymbol{V}-\frac{1}{c^{2}} \frac{\partial^{2} \boldsymbol{V}}{\partial t^{2}}\right)=\left(\nabla^{2}-\frac{1}{c^{2}} \frac{\partial^{2}}{\partial t^{2}}\right)(-i c \Lambda \hat{k}+\boldsymbol{V})=\left(\nabla^{2}-\frac{1}{c^{2}} \frac{\partial^{2}}{\partial t^{2}}\right) V
$$

which we substitute into Equation (20c) to obtain

$$
\nabla(\nabla \cdot V)-\nabla \times(\nabla \times V)=\left(\nabla^{2}-\frac{1}{c^{2}} \frac{\partial^{2}}{\partial t^{2}}+2 i(\hat{k} \cdot \nabla) \frac{1}{c} \frac{\partial}{\partial t}\right) V
$$

Setting

$$
U=V=\nabla \Rightarrow \varphi=\Lambda=-\frac{1}{c^{2}} \frac{\partial}{\partial t}, \quad \boldsymbol{U}=\boldsymbol{V}=\nabla
$$

in the general four-vector dot product in Equation (7b) gives

$$
\nabla \cdot \nabla=\nabla^{2}=\nabla^{2}-\frac{1}{c^{2}} \frac{\partial^{2}}{\partial t^{2}}+2 i(\hat{k} \cdot \nabla) \frac{1}{c} \frac{\partial}{\partial t}
$$

which we substitute into Equation (24e) and reorganize to obtain the four-vector identity

$$
\nabla \times(\nabla \times V)=\nabla(\nabla \cdot V)-\nabla^{2} V
$$

This four-vector identity generalizes the corresponding vector identity in standard three-dimensional Euclidean space [5] [6] given earlier in Equation (19g).

\subsection{Gradient of Dot Product of Four-Vectors: $\nabla(U \cdot V)$}

For general complex four-vectors $U, V$, we have

$$
\begin{aligned}
& U \times(\nabla \times V)=(-i c \varphi \hat{k}+\boldsymbol{U}) \times\left(\boldsymbol{\nabla} \cdot \boldsymbol{V}+i \hat{k} \times\left(\frac{1}{c} \frac{\partial \boldsymbol{V}}{\partial t}+\nabla(c \Lambda)\right)\right) \\
& V \times(\nabla \times U)=(-i c \Lambda \hat{k}+\boldsymbol{V}) \times\left(\boldsymbol{\nabla} \cdot \boldsymbol{U}+i \hat{k} \times\left(\frac{1}{c} \frac{\partial \boldsymbol{U}}{\partial t}+\nabla(c \varphi)\right)\right) \\
& (U \cdot \nabla) V=\left\{\boldsymbol{U} \cdot \nabla+\varphi \frac{\partial}{\partial t}-i \hat{k} \cdot\left(c \varphi \nabla-\boldsymbol{U} \frac{1}{c} \frac{\partial}{\partial t}\right)\right\}(-i c \Lambda \hat{k}+\boldsymbol{V}) \\
& (V \cdot \nabla) U=\left\{\boldsymbol{V} \cdot \nabla+\Lambda \frac{\partial}{\partial t}-i \hat{k} \cdot\left(c \Lambda \nabla-\boldsymbol{V} \frac{1}{c} \frac{\partial}{\partial t}\right)\right\}(-i c \varphi \hat{k}+\boldsymbol{U})
\end{aligned}
$$

Expanding these term by term gives

$$
\begin{aligned}
U & \times(\nabla \times V)+V \times(\nabla \times U) \\
= & -i c \hat{k} \times(\varphi \nabla \times \boldsymbol{V}+\Lambda \nabla \times \boldsymbol{U})+c \hat{k} \times\left\{\hat{k} \times\left(\frac{\varphi}{c} \frac{\partial \boldsymbol{V}}{\partial t}+\frac{\Lambda}{c} \frac{\partial \boldsymbol{U}}{\partial t}\right)\right\} \\
& +c \hat{k} \times\{\hat{k} \times(\varphi \nabla(c \Lambda)+\Lambda \nabla(c \varphi))\}+i\left(\boldsymbol{U} \times\left(\hat{k} \times \frac{1}{c} \frac{\partial \boldsymbol{V}}{\partial t}\right)+\boldsymbol{V} \times\left(\hat{k} \times \frac{1}{c} \frac{\partial \boldsymbol{U}}{\partial t}\right)\right) \\
& +i\{\boldsymbol{U} \times(\hat{k} \times \nabla(c \Lambda))+\boldsymbol{V} \times(\hat{k} \times \nabla(c \varphi))\}+\boldsymbol{U} \times(\nabla \times \boldsymbol{V})+\boldsymbol{V} \times(\boldsymbol{\nabla} \times \boldsymbol{V}) .
\end{aligned}
$$




$$
\begin{aligned}
(U \cdot \nabla) V+(V \cdot \nabla) U \\
=-i c\{(\boldsymbol{U} \cdot \nabla) \Lambda+(\boldsymbol{V} \cdot \nabla) \varphi\} \hat{k}-i c\left(\varphi \frac{\partial \Lambda}{\partial t}+\Lambda \frac{\partial \varphi}{\partial t}\right) \hat{k}-\{c \varphi(\hat{k} \cdot \nabla)(c \Lambda)+c \Lambda(\hat{k} \cdot \nabla)(c \varphi)\} \hat{k} \\
+\left\{\frac{(\hat{k} \cdot \boldsymbol{U})}{c} \frac{\partial(c \Lambda)}{\partial t}+\frac{(\hat{k} \cdot \boldsymbol{V})}{c} \frac{\partial(c \varphi)}{\partial t}\right\} \hat{k}-i c\{\varphi(\hat{k} \cdot \nabla) \boldsymbol{V}+\Lambda(\hat{k} \cdot \nabla) \boldsymbol{U}\} \\
+i\left\{\frac{(\hat{k} \cdot \boldsymbol{U})}{c} \frac{\partial \boldsymbol{V}}{\partial t}+\frac{(\hat{k} \cdot \boldsymbol{V})}{c} \frac{\partial \boldsymbol{U}}{\partial t}\right\}+\left(\varphi \frac{\partial \boldsymbol{V}}{\partial t}+\Lambda \frac{\partial \boldsymbol{U}}{\partial t}\right)+(\boldsymbol{U} \cdot \boldsymbol{\nabla}) \boldsymbol{V}+(\boldsymbol{V} \cdot \boldsymbol{\nabla}) \boldsymbol{U} .
\end{aligned}
$$

We apply three-dimensional Euclidean space vector identities to obtain

$$
\begin{gathered}
c \hat{k} \times\left\{\hat{k} \times\left(\frac{\varphi}{c} \frac{\partial \boldsymbol{V}}{\partial t}+\frac{\Lambda}{c} \frac{\partial \boldsymbol{U}}{\partial t}\right)\right\}=c\left\{\hat{k} \times\left(\frac{\varphi}{c} \frac{\partial \boldsymbol{V}}{\partial t}+\frac{\Lambda}{c} \frac{\partial \boldsymbol{U}}{\partial t}\right)\right\} \hat{k}-c\left(\frac{\varphi}{c} \frac{\partial \boldsymbol{V}}{\partial t}+\frac{\Lambda}{c} \frac{\partial \boldsymbol{U}}{\partial t}\right) \\
c \hat{k} \times\{\hat{k} \times(\varphi \boldsymbol{\nabla}(c \Lambda)+\Lambda \boldsymbol{V}(c \varphi))\}=c\{\hat{k} \times(\varphi \boldsymbol{\nabla}(c \Lambda)+\Lambda \boldsymbol{V}(c \varphi))\} \hat{k}-c(\varphi \boldsymbol{\nabla}(c \Lambda)+\Lambda \boldsymbol{V}(c \varphi)) \\
i\left\{\boldsymbol{U} \times\left(\hat{k} \times \frac{1}{c} \frac{\partial \boldsymbol{V}}{\partial t}\right)+\boldsymbol{V} \times\left(\hat{k} \times \frac{1}{c} \frac{\partial \boldsymbol{U}}{\partial t}\right)\right\}=i\left(\boldsymbol{U} \cdot \frac{1}{c} \frac{\partial \boldsymbol{V}}{\partial t}+\boldsymbol{V} \cdot \frac{1}{c} \frac{\partial \boldsymbol{U}}{\partial t}\right) \hat{k}-i\left\{(\hat{k} \cdot \boldsymbol{U}) \frac{1}{c} \frac{\partial \boldsymbol{V}}{\partial t}+(\hat{k} \cdot \boldsymbol{V}) \frac{1}{c} \frac{\partial \boldsymbol{U}}{\partial t}\right\} \\
i\{\boldsymbol{U} \times(\hat{k} \times \nabla(c \Lambda))+\boldsymbol{V} \times(\hat{k} \times \nabla(c \varphi))\}=i\{\boldsymbol{U} \cdot \boldsymbol{\nabla}(c \Lambda)+\boldsymbol{V} \cdot \boldsymbol{\nabla}(c \varphi)\} \hat{k}-i\{(\hat{k} \cdot \boldsymbol{U}) \nabla(c \Lambda)+(\hat{k} \cdot \boldsymbol{V}) \boldsymbol{\nabla}(c \varphi)\} \\
-i c \hat{k} \times\{\varphi \boldsymbol{\nabla} \times \boldsymbol{V}+\Lambda \boldsymbol{\nabla} \times \boldsymbol{U}\}=-i c\{\varphi \boldsymbol{\nabla}(\hat{k} \cdot \boldsymbol{V})+\Lambda \nabla(\hat{k} \cdot \boldsymbol{U})\}+i c\{\varphi(\hat{k} \cdot \boldsymbol{\nabla}) \boldsymbol{V}+\Lambda(\hat{k} \cdot \boldsymbol{\nabla}) \boldsymbol{U}\}
\end{gathered}
$$

Substituting Equations (24a)-(24e) into Equation (23e), adding the result to Equation (23f) and reorganizing gives

$$
\begin{gathered}
U \times(\nabla \times V)+V \times(\nabla \times U)+(U \cdot \nabla) V+(V \cdot \nabla) U=\left(\frac{i}{c} \frac{\partial}{\partial t} \hat{k}+\nabla\right)(\boldsymbol{U} \cdot \boldsymbol{V})+\left(\frac{i}{c} \frac{\partial}{\partial t} \hat{k}+\nabla\right)\left(-c^{2} \varphi \Lambda\right) \\
+c\left\{\hat{k} \cdot\left(\frac{\varphi}{c} \frac{\partial \boldsymbol{V}}{\partial t}+\frac{\Lambda}{c} \frac{\partial \boldsymbol{U}}{\partial t}\right)\right\} \hat{k}+c\left\{(\hat{k} \cdot \boldsymbol{V}) \frac{1}{c} \frac{\partial \varphi}{\partial t}+(\hat{k} \cdot \boldsymbol{U}) \frac{1}{c} \frac{\partial \Lambda}{\partial t}\right\} \hat{k} \\
-i c\{\varphi \nabla(\hat{k} \cdot \boldsymbol{V})+\Lambda \nabla(\hat{k} \cdot \boldsymbol{U})\} \hat{k}-i c\{(\hat{k} \cdot \boldsymbol{V}) \nabla \varphi+(\hat{k} \cdot \boldsymbol{U}) \nabla \Lambda\} \hat{k} .
\end{gathered}
$$

where we have applied a standard three-dimensional Euclidean space vector identity to introduce

$$
\boldsymbol{\nabla}(\boldsymbol{U} \cdot \boldsymbol{V})=\boldsymbol{U} \times(\boldsymbol{\nabla} \times \boldsymbol{V})+\boldsymbol{V} \times(\boldsymbol{\nabla} \times \boldsymbol{U})+(\boldsymbol{U} \cdot \boldsymbol{\nabla}) \boldsymbol{V}+(\boldsymbol{V} \cdot \boldsymbol{\nabla}) \boldsymbol{U}
$$

Rewriting

$$
\begin{aligned}
& c\left\{\hat{k} \cdot\left(\frac{\varphi}{c} \frac{\partial \boldsymbol{V}}{\partial t}+\frac{\Lambda}{c} \frac{\partial \boldsymbol{U}}{\partial t}\right)\right\} \hat{k}+c\left\{(\hat{k} \cdot \boldsymbol{V}) \frac{1}{c} \frac{\partial \varphi}{\partial t}+(\hat{k} \cdot \boldsymbol{U}) \frac{1}{c} \frac{\partial \Lambda}{\partial t}\right\} \hat{k}=\frac{i}{c} \frac{\partial}{\partial t} \hat{k}\{-i c \hat{k} \cdot(\varphi \boldsymbol{V}+\boldsymbol{U} \Lambda)\} \\
& -i c\{\varphi \nabla(\hat{k} \cdot \boldsymbol{V})+\Lambda \nabla(\hat{k} \cdot \boldsymbol{U})\} \hat{k}-i c\{(\hat{k} \cdot \boldsymbol{V}) \nabla \varphi+(\hat{k} \cdot \boldsymbol{U}) \nabla \Lambda\} \hat{k}=\nabla\{-i c \hat{k} \cdot(\varphi \boldsymbol{V}+\boldsymbol{U} \Lambda)\}
\end{aligned}
$$

we express Equation (24f) in the form

$$
U \times(\nabla \times V)+V \times(\nabla \times U)+(U \cdot \nabla) V+(V \cdot \nabla) U=\left(\frac{i}{c} \frac{\partial}{\partial t} \hat{k}+\nabla\right)\left(\boldsymbol{U} \cdot \boldsymbol{V}-c^{2} \varphi \Lambda-i c \hat{k} \cdot(\varphi \boldsymbol{V}+\boldsymbol{U} \Lambda)\right)
$$

which on substituting the definition of the spacetime derivative four-vector from Equation (6a) and the fourvector dot product obtained in Equation (11c), gives the desired four-vector identity in the final form

$$
\nabla(U \cdot V)=U \times(\nabla \times V)+V \times(\nabla \times U)+(U \cdot \nabla) V+(V \cdot \nabla) U
$$

This four-vector identity generalizes the corresponding vector identity in standard three-dimensional Eucli- 
dean space [5] [6] given earlier in Equation (24g).

\subsection{Triple Cross Product of Four-Vectors: $W \times(U \times V)$}

We introduce a third four-vector defined by

$$
W=-i c \chi \hat{k}+W
$$

and use the four-vector cross product to obtain

$$
W \times(U \times V)=(-i c \chi \hat{k}+W) \times(\boldsymbol{U} \times \boldsymbol{V}-i c \hat{k} \times(\varphi \boldsymbol{V}-\boldsymbol{U} \Lambda))
$$

which we expand as

$$
W \times(U \times V)=-i c \chi \hat{k} \times(\boldsymbol{U} \times \boldsymbol{V})-c^{2} \chi \hat{k} \times\{\hat{k} \times(\varphi \boldsymbol{V}-\boldsymbol{U} \Lambda)\}-i c \boldsymbol{W} \times\{\hat{k} \times(\varphi \boldsymbol{V}-\boldsymbol{U} \Lambda)\}+\vec{W} \times(\boldsymbol{U} \times \boldsymbol{V})
$$

We apply standard three-dimensional Euclidean space vector identities to write

$$
\begin{gathered}
-i c \chi \hat{k} \times(\boldsymbol{U} \cdot \boldsymbol{V})=-i c \chi\{\boldsymbol{U}(\hat{k} \cdot \boldsymbol{V})-\boldsymbol{V}(\hat{k} \cdot \boldsymbol{U})\} \\
-c^{2} \chi \hat{k} \times\{\hat{k} \times(\varphi \boldsymbol{V}-\boldsymbol{U} \Lambda)\}=-c^{2} \chi\{\hat{k}(\hat{k} \cdot(\varphi \boldsymbol{V}-\boldsymbol{U} \Lambda))-(\varphi \boldsymbol{V}-\boldsymbol{U} \Lambda)\} \\
-i c \boldsymbol{W} \times\{\hat{k} \times(\varphi \boldsymbol{V}-\boldsymbol{U} \Lambda)\}=-i c\{\hat{k}(\boldsymbol{W} \cdot(\varphi \boldsymbol{V}-\boldsymbol{U} \Lambda))-(\varphi \boldsymbol{V}-\boldsymbol{U} \Lambda)(\hat{k} \cdot \boldsymbol{W})\} \\
\boldsymbol{W} \times(\boldsymbol{U} \times \boldsymbol{V})=\boldsymbol{U}(\boldsymbol{W} \cdot \boldsymbol{V})-\boldsymbol{V}(\boldsymbol{W} \cdot \boldsymbol{U})
\end{gathered}
$$

which we substitute into Equation (28b) and collect like terms to obtain

$$
\begin{aligned}
W \times(U \times V)= & \boldsymbol{U}\left(\boldsymbol{W} \cdot \boldsymbol{V}-c^{2} \chi \Lambda-i c \hat{k} \cdot(\chi \boldsymbol{V}+\boldsymbol{W} \Lambda)\right)-\boldsymbol{V}\left(\boldsymbol{W} \cdot \boldsymbol{U}-c^{2} \chi \varphi-i c \hat{k} \cdot(\chi \boldsymbol{U}+\boldsymbol{W} \varphi)\right) \\
& -i c \varphi \hat{k}(\boldsymbol{W} \cdot \boldsymbol{V}-i c \hat{k} \cdot \chi \boldsymbol{V})+i c \Lambda \hat{k}(\boldsymbol{W} \cdot \boldsymbol{U}-i c \hat{k} \cdot \chi \boldsymbol{U}) .
\end{aligned}
$$

We rewrite the last two terms in Equation (28g) by subtracting and adding appropriate terms according to

$$
\begin{aligned}
& \boldsymbol{W} \cdot \boldsymbol{V}-i c \hat{k} \cdot \chi \boldsymbol{V}=\left(\boldsymbol{W} \cdot \boldsymbol{V}-c^{2} \chi \Lambda-i c \hat{k} \cdot \chi \boldsymbol{V}-i c \hat{k} \cdot \boldsymbol{W} \Lambda\right)+c^{2} \chi \Lambda+i c \hat{k} \cdot \boldsymbol{W} \Lambda \\
& \boldsymbol{W} \cdot \boldsymbol{U}-i c \hat{k} \cdot \chi \boldsymbol{U}=\left(\boldsymbol{W} \cdot \boldsymbol{U}-c^{2} \chi \varphi-i c \hat{k} \cdot \chi \boldsymbol{U}-i c \hat{k} \cdot \boldsymbol{W} \varphi\right)+c^{2} \chi \varphi+i c \hat{k} \cdot \boldsymbol{W} \varphi
\end{aligned}
$$

which we substitute back and collect like terms to express Equation (28g) in the form

$$
\begin{aligned}
W & \times(U \times V) \\
& =(-i c \varphi \hat{k}+\boldsymbol{U})\left(\boldsymbol{W} \cdot \boldsymbol{V}-c^{2} \chi \Lambda-i c \hat{k} \cdot(\chi \boldsymbol{V}+\boldsymbol{W} \Lambda)\right)-(-i c \Lambda \hat{k}+\boldsymbol{V})\left(\boldsymbol{W} \cdot \boldsymbol{U}-c^{2} \chi \varphi-i c \hat{k} \cdot(\chi \boldsymbol{U}+\boldsymbol{W} \varphi)\right) .
\end{aligned}
$$

Finally, we apply the usual definitions of the four-vectors $U, V$ and introduce the four-vector dot products

$$
W \cdot V=\boldsymbol{W} \cdot \boldsymbol{V}-c^{2} \chi \Lambda-i c \hat{k} \cdot(\chi \boldsymbol{V}+\boldsymbol{W} \Lambda), \quad W \cdot U=\boldsymbol{W} \cdot \boldsymbol{U}-c^{2} \chi \varphi-i c \hat{k} \cdot(\chi \boldsymbol{U}+\boldsymbol{W} \varphi)
$$

in Equation (29c) to obtain the desired four-vector identity in the form

$$
W \times(U \times V)=U(W \cdot V)-V(W \cdot U)
$$

This four-vector identity generalizes the corresponding vector identity in standard three-dimensional Euclidean space given earlier in Equation (28f).

We easily apply the four-vector identity obtained in Equation (30a) to establish the cyclic property of the triple four-vector cross product in the form

$$
W \times(U \times V)+U \times(V \times W)+V \times(W \times U)=0
$$

which generalizes the corresponding vector identity in standard three-dimensional Euclidean space [5] [6].

The four-vector identities derived in Equations (12), (14), (15g), (16d), (18d), (22), (26), (30a) and (30b) con- 
firm the consistency of the definitions of the complex four-component vectors and corresponding mathematical operations within the complex four-dimensional spacetime frame. This means that complex four-dimensional spacetime frame characterized by complex four-component vectors is a consistent mathematical extension of the standard three-dimensional space characterized by the usual three-component vectors. The other four-vector identities can be derived following similar procedure.

\section{Contravariant and Covariant Four-Vectors}

To complete the mathematical formalism within complex four-dimensional spacetime frame, we introduce contravariant and covariant forms, which are useful in carrying out general mathematical operations with four-vectors. A contravariant four-vector is specified by positive spatial components, while a covariant four-vector is specified by negative spatial components. We represent the four-vector $V$ in a contravariant form by $V^{\mu}$ and in a covariant form by $V_{\mu}$, where $\mu=0,1,2,3$ with 0 labeling the temporal component, while 1, 2, 3 label the spatial $(x, y, z)$ components, respectively. We define $V^{\mu}$ and $V_{\mu}$ below.

Denoting the four unit vectors by $\hat{k}, \hat{x}, \hat{y}, \hat{z}$ as presented in this paper, we define the contravariant coordinates $x^{\mu}$ of the general four-dimensional complex spacetime frame in the form [7] [8]

$$
x^{\mu}, \quad \mu=0,1,2,3, \quad x^{0}=i c t, \quad x^{1}=x, \quad x^{2}=y, \quad x^{3}=z
$$

The corresponding covariant coordinates $x_{-}$are defined in the form

$$
x_{\mu}, \quad \mu=0,1,2,3, \quad x_{0}=x^{0}=i c t, \quad x_{1}=-x^{1}=-x, \quad x_{2}=-x^{2}=-y, \quad x_{3}=-x^{3}=-z
$$

We then express the contravariant spacetime displacement four-vector $X^{\mu}$ and the corresponding covariant form $X_{\mu}$ as

$$
X^{\mu}=-x^{0} \hat{k}+x^{1} \hat{x}+x^{2} \hat{y}+x^{3} \hat{z}, \quad X_{\mu}=-x_{0} \hat{k}+x_{1} \hat{x}+x_{2} \hat{y}+x_{3} \hat{z}
$$

which we introduce the position vector $\boldsymbol{r}=x \hat{x}+y \hat{y}+z \hat{z}$ according to Equations (31a)-(31b) to express in the final forms

$$
X^{\mu}=-(i c t \hat{k}-\boldsymbol{r}), \quad X_{\mu}=-(i c t \hat{k}+\boldsymbol{r})
$$

The spacetime event interval takes the contravariant and covariant forms $\left(\mathrm{d} x^{0}=\mathrm{d} x_{0}\right)$

$$
\mathrm{d} X^{\mu}=-\mathrm{d} x_{0} \hat{k}+\mathrm{d} \boldsymbol{r}=-(i c \mathrm{~d} t \hat{k}-\mathrm{d} \boldsymbol{r}), \quad \mathrm{d} X_{\mu}=-\mathrm{d} x_{0} \hat{k}-\mathrm{d} \boldsymbol{r}=-(i c \mathrm{~d} t \hat{k}+\mathrm{d} \boldsymbol{r})
$$

A general four-vector $V$ as defined earlier is expressed in contravariant and covariant forms according to

$$
\begin{array}{ccc}
V^{0}=i c \phi, \quad V^{1}=V_{x}, \quad V^{2}=V_{y}, & V^{3}=V_{z} \\
V_{0}=V^{0}, \quad V_{1}=-V^{1}, & V_{1}=-V^{1}, \quad V_{3}=-V^{3}
\end{array}
$$

with

$$
V^{\mu}=-V^{0} \hat{k}+V^{1} \hat{x}+V^{2} \hat{y}+V^{3} \hat{z}, \quad V_{\mu}=-V_{0} \hat{k}+V_{1} \hat{x}+V_{2} \hat{y}+V_{3} \hat{z}
$$

which we express in the final forms

$$
V^{\mu}=-(i c \phi \hat{k}-\boldsymbol{V}), \quad V_{\mu}=-(i c \phi \hat{k}+\boldsymbol{V})
$$

The contravariant and covariant four-vectors are related through complex conjugation in the form

$$
V^{\mu *}=-V_{\mu}, \quad V_{\mu}^{*}=-V^{\mu}
$$

We use this contravariant-covariant four-vector conjugation relation to obtain

$$
V^{\mu} \cdot V_{\mu}^{*}=-\left(V^{\mu}\right)^{2}, \quad V^{\mu *} \cdot V_{\mu}=-\left(V_{\mu}\right)^{2}
$$

which provides the definition of the invariant length $\bar{V}$ of the general four-vector $V^{\mu}$ or $V_{\mu}$ according to

$$
\bar{V}^{2}=\left|-\left(V^{\mu}\right)^{2}\right|=\left|-\left(V_{\mu}\right)^{2}\right|
$$


We express this in the general form

$$
\bar{V}^{2}=\left|V^{\mu} \cdot V_{\mu}^{*}\right|=\left|V^{\mu *} \cdot V_{\mu}\right|
$$

Using $V^{\mu}, V_{\mu}$ from Equation (32d), noting the relation in Equation (32e), we apply Equation (32g) or (32h) to obtain the invariant length in the explicit form

$$
\bar{V}^{2}=\sqrt{1+\frac{(2 c \phi \hat{k} \cdot \boldsymbol{V})^{2}}{\left(c^{2} \phi^{2}-|\boldsymbol{V}|^{2}\right)^{2}}}\left(c^{2} \phi^{2}-|\boldsymbol{V}|^{2}\right)=\eta\left(c^{2} \phi^{2}-|\boldsymbol{V}|^{2}\right)
$$

which is modified by a factor $\eta$ arising from the general orientation of the temporal unit vector relative to the spatial unit vectors according to the definition

$$
\eta=\sqrt{1+\frac{(2 c \phi \hat{k} \cdot \boldsymbol{V})^{2}}{\left(c^{2} \phi^{2}-|\boldsymbol{V}|^{2}\right)^{2}}}, \quad|\boldsymbol{V}|^{2}=\boldsymbol{V} \cdot \boldsymbol{V}
$$

The invariant length ds of the spacetime event interval is then modified according to

$$
(\mathrm{d} s)^{2}=\eta\left((c \mathrm{~d} t)^{2}-(\mathrm{d} \boldsymbol{r})^{2}\right), \quad \eta=\sqrt{1+\frac{4(\hat{k} \cdot \boldsymbol{v})^{2}}{c^{2}\left(1-\frac{v^{2}}{c^{2}}\right)^{2}}}, \quad \boldsymbol{v}=\frac{\mathrm{d} \boldsymbol{r}}{\mathrm{d} t}
$$

where $\boldsymbol{v}$ is the velocity, with speed $v=|\boldsymbol{v}|$ defined as usual. This result has important implications for current theoretical and experimental research in physics [9] [10].

\section{Tensors in the Complex Spacetime Frame}

We now develop the procedure for defining tensors [7] [8] within the general four-dimensional complex spacetime frame. To put the presentation in familiar form, we adopt the standard contravariant and covariant fourvector notation to express $V^{\mu}$ and $V_{\mu}$ from Equation (20d) in the form

$$
V^{\mu}=-(i c \phi,-\boldsymbol{V}) ; \quad V_{\mu}=-(i c \phi, \boldsymbol{V})
$$

with complex conjugates taking the form

$$
V^{\mu *}=(i c \phi, \boldsymbol{V})=-V_{\mu} ; \quad V_{\mu}^{*}=(i c \phi,-\boldsymbol{V})=-V^{\mu}
$$

where the usual four-vector mathematics is applied, but now taking account of the general orientation of the temporal unit vector $\hat{k}$ relative to the spatial unit vectors $\hat{x}, \hat{y}, \hat{z}$ to obtain the general results presented above.

Using the complex conjugation relation from Equation (33b) gives

$$
V^{\mu} \cdot V_{v}^{*}=-V^{\mu} \cdot V^{v} ; \quad V^{\mu *} \cdot V_{v}=-V_{\mu} \cdot V_{v}
$$

from which a definition of complex contravariant and covariant rank-2 tensors $T^{\mu v}$ and $T_{\mu v}$ follows according to

$$
T^{\mu \nu}=-V^{\mu} \cdot V_{v}^{*}=V^{\mu} \cdot V^{\nu} ; \quad T_{\mu v}=-V^{\mu *} \cdot V_{v}=V_{\mu} \cdot V_{v}
$$

In addition,

$$
V^{\mu} \cdot V^{\nu *}=-V^{\mu} \cdot V_{v} ; \quad V^{\mu *} \cdot V^{\nu}=-V_{\mu} \cdot V^{v}
$$

provides a definition of complex rank-2 mixed tensors $T_{v}^{\mu}$ and $T_{\mu}^{v}$ in the form

$$
T_{v}^{\mu}=-V^{\mu} \cdot V^{\nu *}=V^{\mu} \cdot V_{v} ; \quad T_{\mu}^{v}=-V^{\mu *} \cdot V^{v}=V_{\mu} \cdot V^{v}
$$


The definition of more general tensors of higher rank follows easily. Some mathematical properties of the rank-2 tensors defined above can be obtained by interchanging the indices $\mu, v$ or taking complex conjugation or carrying out both operations simultaneously.

The complete definition of contravariant and covariant complex four-vectors, which can be used to define tensors of general ranks in contravariant, covariant or mixed forms, provides the necessary foundation for more general vector and tensor analysis, leading to reformulation of differential geometry a complex four-dimensional spacetime frame. This is indeed the origin of a new framework for studying physics, mathematics and related disciplines in the 21st-century and beyond. Some important implications for physics are presented in [1].

\section{Conclusion}

All the basic four-vector identities which we have derived in this work take exactly the same form as the standard vector identities established in the familiar three-dimensional space. This confirms the consistency of the definition of complex four-component vectors and corresponding mathematical operations within a complex four-dimensional spacetime frame with an imaginary temporal axis. The contravariant and covariant forms introduced here lead to consistent definitions of complex tensors, which are the basic quantities for reformulation of differential geometry within complex spacetime frame. This new mathematical framework has important implications for various models of relativistic mechanics, quantum field theory and general relativity as a theory of gravitation and cosmology.

\section{Acknowledgements}

I thank Maseno University, Kenya, for supporting this work by providing facilities and working environment.

\section{References}

[1] Akeyo Omolo, J. (2014) On a Derivation of the Temporal Unit Vector of Space-Time Frame. Journal of Applied Mathematics and Physics, in Press.

[2] Poincare, H. (1906) Sur la dynamique de l'e'lectron (On the Dynamics of the Electron). Rendiconti del Circolo Matematico di Palermo, 21, 129.

[3] Lorentz, H.A. (1904) Electromagnetic Phenomena in a System Moving with Any Velocity Smaller than That of Light. Proceedings of the Royal Netherlands Academy of Arts and Sciences, 6, 809

[4] Einstein, A. (2003) The Meaning of Relativity. Routledge Classics, London and New York.

[5] Charbonneau, P. (2013) Solar and Stellar Dynamics. Saas-Fee Advanced Course, 39, 215.

[6] Arfken, G.B. and Weber, H.J. (1995) Mathematical Methods for Physicists. Academic Press Inc., San Diego.

[7] Landau, L.D. and Lifshitz, E.M. (1975) The Classical Theory of Fields. Pergamon Press Ltd., Oxford.

[8] Dirac, P.A.M. (1975) General Theory of Relativity. John Wiley and Sons Inc., New York.

[9] Bogoslovsky, G.Y. (2007) Some Physical Displays of the Space Anisotropy Relevant to the Feasibility of Its Being Detected at a Laboratory. arXiv:0706.2621 [gr-qc]

[10] Gibbons, G.W., Gomis, J. and Pope, C.N. (2007) General Very Special Relativity in Finsler Geometry. Physical Review D, 76, Article ID: 081701. http://dx.doi.org/10.1103/PhysRevD.76.081701 
Scientific Research Publishing (SCIRP) is one of the largest Open Access journal publishers. It is currently publishing more than 200 open access, online, peer-reviewed journals covering a wide range of academic disciplines. SCIRP serves the worldwide academic communities and contributes to the progress and application of science with its publication.

Other selected journals from SCIRP are listed as below. Submit your manuscript to us via either submit@scirp.org or Online Submission Portal.
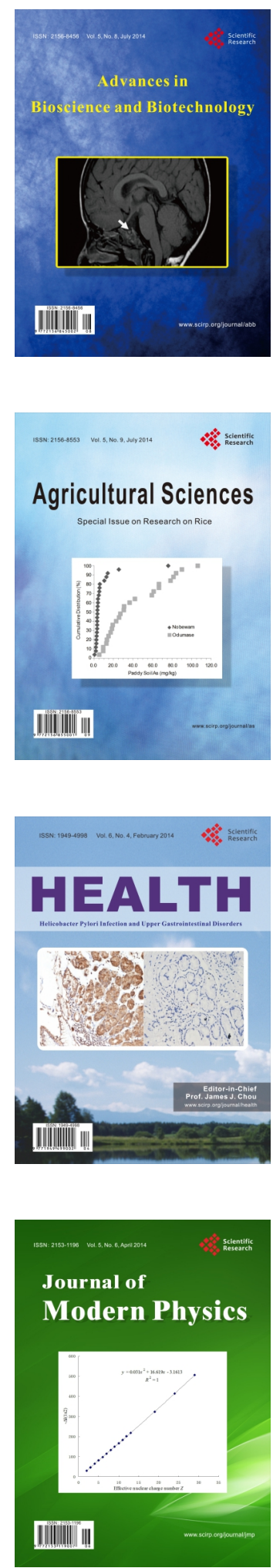
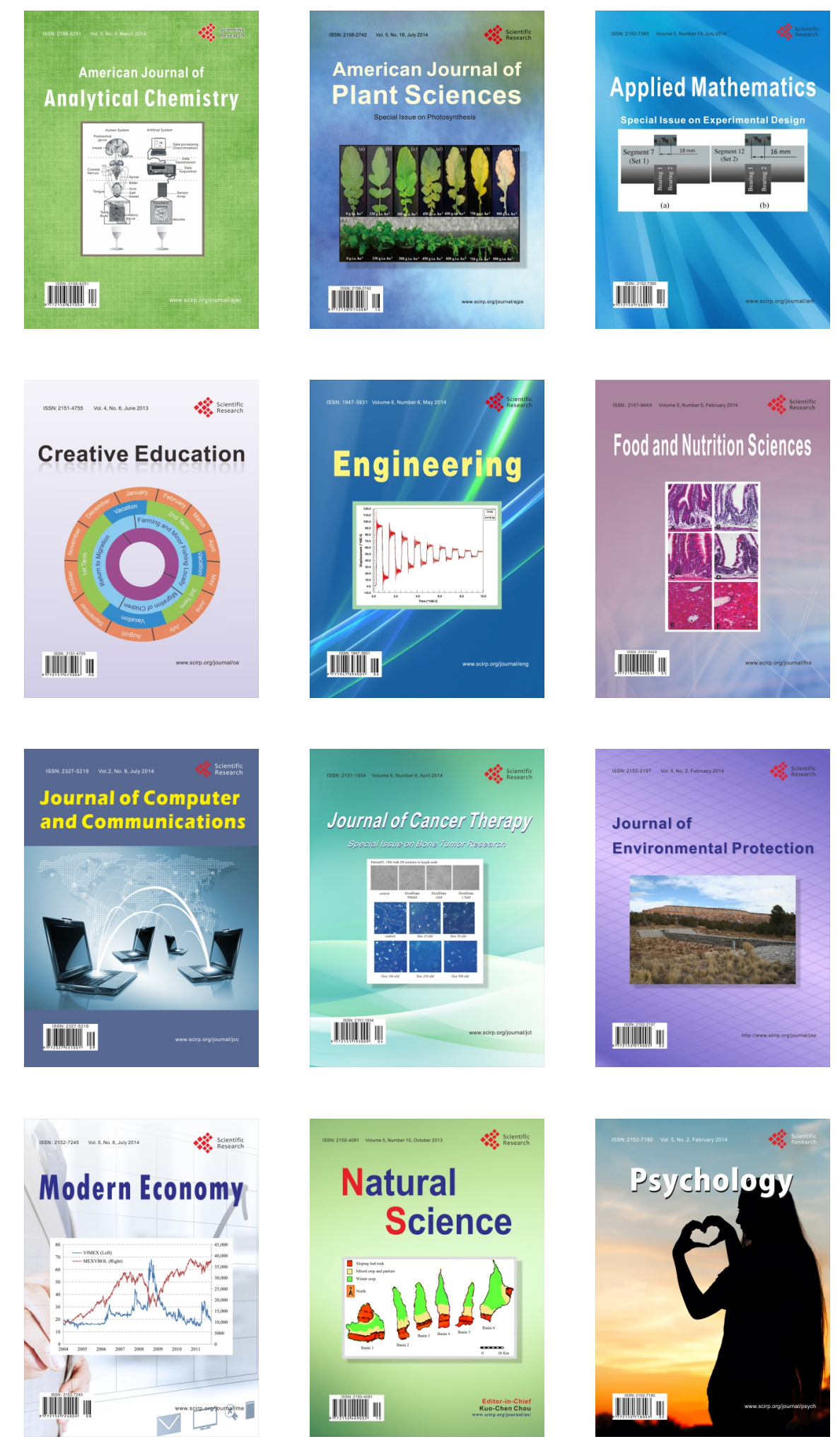